\title{
Fluid Dynamic Forces on Plunging Spanwise-Flexible Elliptical Flat Plates at Low Reynolds Numbers
}

\author{
Jonathan M. Rausch ${ }^{1}$, Luis P. Bernal ${ }^{2}$, Carlos S. Cesnik ${ }^{3}$, \\ Department of Aerospace Engineering, University of Michigan, Ann Arbor, MI, 48109 \\ Wei Shyy ${ }^{4}$, \\ Department of Aerospace Engineering, University of Michigan, Ann Arbor, MI, 48109 \\ Department of Mechanical Engineering, Hong Kong University of Science and Technology, Kowloon, Hong Kong \\ and Lawrence Ukeiley ${ }^{5}$ \\ Department of Mechanical and Aerospace Engineering, University of Florida, Shalimar, FL, 32579
}

We consider the aerodynamic performance of flexible isotropic elliptical wings undergoing periodic plunge motions. Experiments were conducted in the Low Turbulence Water Channel at the University of Michigan using a pitch-plunge apparatus. Experimental results include dye flow visualization, laser Doppler vibrometer (LDV) wing deformation measurements, particle image velocimetry (PIV) flowfield quantification, and direct force measurements establishing a novel experimental framework for investigating pitchingplunging and flapping flexible wings. This investigation focuses on the effect of wing stiffness parameter, $\Pi_{1}$, defined as the ratio of elastic to fluid dynamic forces. A parameter sweep is performed that spans four orders of magnitude from order $10^{1}$ to $10^{4}$. The $\Pi_{1}$-parameter is varied by changing the plate thickness and material properties. The effects of structural density to fluid density, $\bar{\rho}$, and the thickness to chord ratio, $\bar{h}_{s}$, are shown to be small. The wings have elliptical planform with aspect ratio 6.1. Sinusoidal plunging kinematics are used in forward fight at a low Reynolds number $(5,300)$ with a neutral mean effective angle of attack. The plunging motion has large reduced frequency (1.82) and modest chordnormalized plunge amplitude (0.175). Deformation measurements show that for the present conditions the wings bend without twisting. PIV measurements at the 50\%- and $75 \%$ spanwise locations show that large deflections at the wing tip result in a stronger outboard leading edge vortex due to the increased effective angle of attack for increased flexibility. The force measurements proved that the prescribed parametric configuration is not thrust producing due to small wing cross-sectional thickness, motion kinematics, and lack of aerodynamic-twist. The lift (normal) force coefficient for moderate wing stiffness parameter $\left(\Pi_{1}\right.$ of order $\left.10^{2}\right)$ is larger compared to the rigid wing results. The most flexible wing produced a lift coefficient history below the rigid wing and lags the rigid wing phase. For the rigid cases the force measurements show good agreement with quasi-steady two-dimensional potential flow theory, suggesting that for the present conditions tip vortex effects are small.

\section{Nomenclature}

\footnotetext{
${ }^{1}$ Graduate Research Assistant, Department of Aerospace Engineering, AIAA Student Member, rauschjm@umich.edu

${ }^{2}$ Associate Professor, AIAA Member, lpb@umich.edu.

${ }^{3}$ Professor, Department of Aerospace Engineering, University of Michigan, AIAA Associate Fellow

${ }^{4}$ Professor Clarence L. "Kelly" Johnson Collegiate Professor, Department of Aerospace Engineering, University of Michigan; currently, Provost \& Chair Professor, Department of Mechanical Engineering, Hong Kong University of Science and Technology, AIAA Fellow, AIAA Fellow

${ }^{5}$ Assistant Professor, AIAA Associate Fellow
} 


\begin{tabular}{|c|c|c|}
\hline$b$ & span & {$[\mathrm{m}]$} \\
\hline$c$ & root chord & {$[\mathrm{m}]$} \\
\hline$C_{L}$ & total lift coefficient, $C_{L}=L / q S$ & {$[1]$} \\
\hline$C_{l}$ & lift coefficient per unit span & {$[1 / \mathrm{m}]$} \\
\hline$D$ & flat plate bending stiffness, $D=E t^{3} / 12\left(1-v^{2}\right)$ & {$[\mathrm{N} \cdot \mathrm{m}]$} \\
\hline E & modulus of elasticity & {$[\mathrm{Pa}]$} \\
\hline$F$ & force & {$[\mathrm{N}]$} \\
\hline$f$ & frequency & {$[\mathrm{Hz}]$} \\
\hline$h$ & plunge amplitude of oscillation & {$[\mathrm{m}]$} \\
\hline $\bar{h}$ & reduced plunge amplitude of oscillation & {$[1]$} \\
\hline$h_{s}$ & structural thickness & {$[\mathrm{m}]$} \\
\hline $\bar{h}_{s}$ & reduced structural thickness & {$[1]$} \\
\hline$k$ & reduced frequency, $k=\pi f c / U_{\infty}$ & {$[1]$} \\
\hline$L$ & total lift force & {$[\mathrm{N}]$} \\
\hline$R e$ & Reynolds number, $R e=c U_{\infty} / v$ & [1] \\
\hline$S$ & planform area & {$\left[\mathrm{m}^{2}\right]$} \\
\hline$S t$ & Strouhal number, $S t=2 f h_{o} / U_{\infty}=2 k h_{o} / \pi$ & {$[1]$} \\
\hline$U$ & velocity & {$[\mathrm{m} / \mathrm{s}]$} \\
\hline $\mathrm{x}$ & streamwise coordinate & [m] \\
\hline $\mathrm{y}$ & spanwise coordinate & {$[\mathrm{m}]$} \\
\hline $\mathrm{z}$ & plunge/normal coordinate & {$[\mathrm{m}]$} \\
\hline \multicolumn{3}{|c|}{$\underline{\text { Greek Symbols }}$} \\
\hline$\alpha_{e f f}$ & effective angle of attack & [deg.] \\
\hline$\alpha_{0}$ & mean angle of attack & [deg.] \\
\hline$v$ & Poisson's ratio & {$[1]$} \\
\hline$\Pi_{1}$ & effective stiffness, $\Pi_{1}=D /\left(\rho_{\infty} U_{\infty}^{2} c^{3}\right)$ & {$[1]$} \\
\hline$\mu$ & dynamic viscosity of fluid & {$[\mathrm{Pa} \mathrm{s}]$} \\
\hline$\rho$ & density & {$\left[\mathrm{kg} / \mathrm{m}^{3}\right]$} \\
\hline $\bar{\rho}$ & structure to fluid density ratio & [1] \\
\hline$\phi$ & positive phase offset for pitch relative to plunge & [rad.] \\
\hline$\omega$ & vorticity & {$[1 / \mathrm{s}]$} \\
\hline$\omega^{*}$ & normalized vorticity, $\omega^{*}=\omega c / U_{\infty}$ & {$[1]$} \\
\hline \multicolumn{3}{|c|}{$\underline{\text { Subscripts }}$} \\
\hline$\infty$ & freestream condition & \\
\hline$f$ & fluid designation & \\
\hline$s$ & structure designation & \\
\hline
\end{tabular}

\section{Introduction}

The aerodynamics of pitching-plunging and flapping flexible-wings are of interest for application to Micro Air Vehicle (MAV) design. Nature presents many examples of small insects and birds with high wing flexibilities that have unique capabilities to maneuver, respond to gusts and perch yet engineering a MAV with comparable performance remains elusive. An understanding of how flexibility garners biological flyers beneficial flight performance is needed. For engineers to take advantage of biological flyer's optimal flight performance ${ }^{1,2}$ computational and experimental frameworks along with appropriate scaling parameters must be developed that capture the fluid and structural dynamics of flapping flexible-wings.

Studies of flexibility have focused on the beneficial mechanisms resulting from the introduction of chordwise $^{3,4,5,6,7,8}$ and spanwise ${ }^{9,10}$ flexibility. These studies show how flexibility can be used to improve 
aerodynamic and propulsion performance. Of particular importance are earlier investigations of the effect of chordwise and spanwise wing flexibility on the aerodynamic and propulsion performance of flapping airfoil-wings reported by Heathcote and co-workers $3,9,11$. They found that moderate amounts of flexibility improves lift and thrust performance. In a recent study Rausch et al. ${ }^{12}$ used laser Doppler vibrometer and PIV measurements to determine the spanwise deformation and flow field of an elliptical wing in pure plunging motion. The results showed that the large deformation of the wing produced large effective angle of attack near the wing tip and as a result the leading edge vortex strength increased near the wing tip. These observations are consistent with Heathcote and co-workers' results.

The present study expands the earlier investigation of Rausch, et al., which included four wings with $\Pi_{1}$ of 30 , $1,430,2,030$, and 91,300 and were experimentally characterized with dye flow visualization, PIV, and LDV. The main conclusions of that study were:

* $\quad \Pi_{1}$ values of 1,430 and above were essentially rigid and the $\Pi_{1}$ value of 30 was highly flexible.

* Flow topology was influenced by the increased effective angle of attack at outboard span location for the flexible wing. The effective angle of attack increase resulted in a significantly larger leading edge vortex at $75 \%$ span location than the $50 \%$ location.

* The LDV indicated that the there was negligible aerodynamic-twist for the flexible wing

The present paper includes LDV deformation measurements and PIV for $\Pi_{1}$ of order $10^{2}$, and direct force measurements for these cases and all the cases reported by Rausch, et al.. We consider pure-plunge kinematics in a forward flight condition and study the flow at low Reynolds number $(5,300)$ and high reduced frequency $(1.83)$ for modest chord normalized plunge amplitude (0.175).

Also of particular interest is the development of leading edge, trailing edge and tip vortices. Many studies discussed by Shyy et al $2^{, 5}$ have documented the formation and evolution of these vortical structures and the role they play in force production. However, the relative importance of vortical structure and flow inertia at high frequency remains unresolved. The recent investigations by $\mathrm{Ol} \mathrm{et} \mathrm{al.}{ }^{13}$ and Baik et al. ${ }^{14}$ show that very large force coefficients develop in high-pitch-rate perching maneuvers. A large fraction of the force is due to non-circulatory effects. Furthermore it was found in $\mathrm{Ol}$ et al. that the wing aspect ratio had a relatively small impact on aerodynamic performance.

\section{Scaling Parameters}

The scaling parameters are derived from the relevant physical parameters listed in Table 1.

Table 1. Table of physical parameters

\begin{tabular}{lcl}
\hline \hline fluid density & $\rho_{f}$ & {$\left[\mathrm{~kg} / \mathrm{m}^{3}\right]$} \\
fluid viscosity & $\mu$ & {$[\mathrm{kg} / \mathrm{m} \mathrm{s}]$} \\
freestream velocity & $U_{\infty}$ & {$[\mathrm{m} / \mathrm{s}]$} \\
half span & $b$ & {$[\mathrm{~m}]$} \\
root chord & $c$ & {$[\mathrm{~m}]$} \\
thickness & $t$ & {$[\mathrm{~m}]$} \\
structural density & $\rho_{s}$ & {$\left[\mathrm{~kg} / \mathrm{m}^{3}\right]$} \\
Young's modulus & $E$ & {$\left[\mathrm{~kg} / \mathrm{ms}^{2}\right]$} \\
Poisson's ratio & $v$ & {$[1]$} \\
plunge amplitude & $h$ & {$[\mathrm{~m}]$} \\
plunge frequency & $f$ & {$[1 / \mathrm{s}]$} \\
geometric angle of attack & $\alpha$ & {$[\mathrm{rad}]$.} \\
fluid dynamic force & $F$ & {$\left[\mathrm{~kg} \mathrm{~m} / \mathrm{s}^{2}\right]$} \\
\hline \hline
\end{tabular}

The physical parameters in Table 1 are a combination of thirteen flow, structural, and kinematic parameters with three dimensions. Using the fluid density, flow velocity, and chord as the basis variables in a dimensional analysis $^{15,16}$ yields 10 non-dimensional scaling parameters that are listed in Table 2 . The non-dimensional parameters can also be derived from the kinematics and governing equations for the fluid and structure ${ }^{5,6}$. 
Table 2. Non-dimensional quantities with descriptions of their significance

\begin{tabular}{|c|c|c|}
\hline Non-dimensional Quantity & Definition & Description \\
\hline Reynolds number & $R e=\frac{\rho_{\infty} U_{\infty} c}{\mu}$ & ratio of inertial to viscous forces \\
\hline Aspect ratio & $A R=\frac{2 b}{c}$ & span normalized with the chord \\
\hline Thickness to chord ratio & $\bar{h}_{s}=\frac{h_{s}}{c}$ & structural thickness normalized with the chord \\
\hline Density ratio & $\bar{\rho}=\frac{\rho_{s}}{\rho_{f}}$ & ratio of structural density to fluid density \\
\hline Poisson ratio & $v$ & axial and transverse strain relationship \\
\hline Effective stiffness & $\Pi_{1}=\frac{D}{\rho_{\infty} U_{\infty}^{2} c^{3}}$ & ratio between elastic and fluid dynamic forces \\
\hline Reduced frequency & $k=\frac{\pi f c}{U_{\infty}}$ & $\begin{array}{l}\text { compares spatial wavelength of the flow disturbance with the } \\
\text { chord }\end{array}$ \\
\hline Strouhal number & $S t=\frac{2 f h}{U_{\infty}}$ & ratio of plunging speed to freestream velocity \\
\hline Angle of attack & $\alpha$ & $\begin{array}{l}\text { Curvature of the streamlines leading to pressure changes on } \\
\text { the wing surface }\end{array}$ \\
\hline Force coefficient & $C_{F}=\frac{F}{0.5 \rho_{f} U_{\infty}^{2} S}$ & $\begin{array}{l}\text { fluid dynamic force normalized with dynamic pressure and the } \\
\text { wing surface area }\end{array}$ \\
\hline
\end{tabular}

For this study the effective stiffness, $\Pi_{1}$, is varied over a large range while all other scaling parameters are held nearly constant with small variation in structural thickness and structural density to facilitate the change in effective stiffness. The effective stiffness is the ratio of the equivalent flat plate stiffness to the aerodynamic loading.

\section{Study Parameters}

The focus of the parametric study is the effect of spanwise flexibility on the fluid dynamics of plunging linearly elastic homogeneous isotropic wings. By fixing the kinematics and Reynolds number the effect of flexibility is investigated. The details of the experimental investigation along with the scaling parameters will follow.

The sinusoidal plunging motion is characterized by a normalized plunge of 0.175 and a Strouhal number of 0.203. This Strouhal number lies within the range of Strouhal numbers $(0.2<S t<0.4)$ used by natural flapping wing flyers ${ }^{17}$. This Strouhal number range has been found to provide high propulsive efficiencies for spanwiseflexible wings undergoing a sinusoidal plunging motion. The physical quantities for plunge amplitude, velocity and acceleration are plotted in Figure 1. The motion begins with a positive position and oscillates with the cosine. 


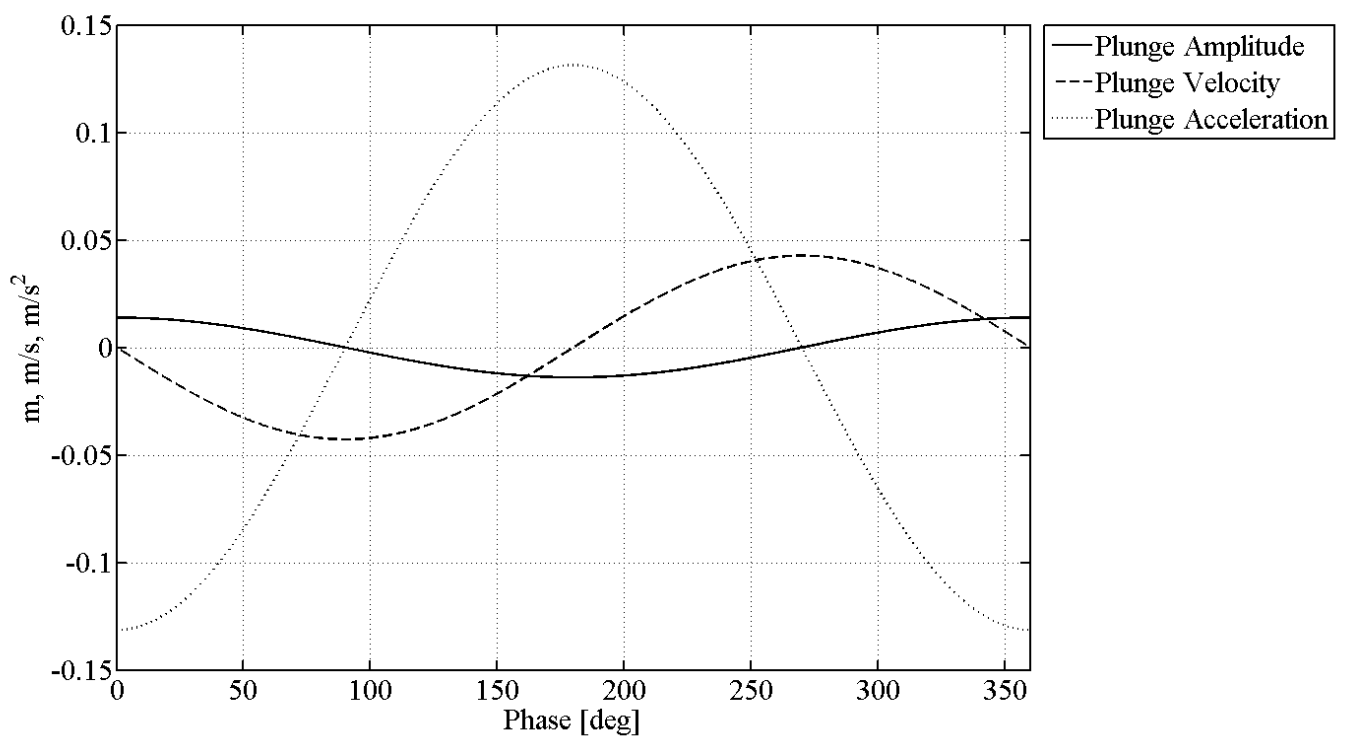

Figure 1. Plot of plunge amplitude, velocity, and acceleration

A summary of the physical and non-dimensional kinematic parameters is provided in Table 3.

Table 3. Physical and non-dimensional kinematic parameters

\begin{tabular}{ll||ll}
\hline \hline Physical Parameters & & Non-dimensional Parameters & \\
$f[\mathrm{~Hz}]$ & 0.489 & $R e$ & 5,300 \\
$h[\mathrm{~mm}]$ & 13.8 & $k$ & 1.82 \\
$U_{\infty}[\mathrm{mm} / \mathrm{s}]$ & 67 & $S t$ & 0.203 \\
$c[\mathrm{~mm}]$ & 79.4 & $\bar{h}$ & 0.175 \\
& & $\alpha_{0}$ & 0 \\
\hline \hline
\end{tabular}

This study uses six elliptical-planform flat-plate wing models with square edges. Three models are made of aluminum and three are made of high-density polyethylene (HDPE). There are four plate thicknesses that are used in combination with the two materials to yield a range of $\Pi_{1}$ from 30 to 91,300 .

Table 4 summarizes the structural properties of the wings used.

Table 4. Structural properties

\begin{tabular}{|c|c|c|c|c|c|}
\hline Wing Geometry & & Aluminum Wing & & HDPE Wing & \\
\hline$b[\mathrm{~mm}]$ & 241.3 & $E \quad\left[\mathrm{~kg} / \mathrm{ms}^{2}\right]$ & $70 \times 10^{9}$ & $E \quad\left[\mathrm{~kg} / \mathrm{ms}^{2}\right]$ & $1.5 \times 10^{9}$ \\
\hline$c[\mathrm{~mm}]$ & 79.4 & $v$ & 0.3 & $v$ & 0.35 \\
\hline$A R$ & 7.65 & $\rho \quad\left[\mathrm{kg} / \mathrm{m}^{3}\right]$ & 2,700 & $\rho \quad\left[\mathrm{kg} / \mathrm{m}^{3}\right]$ & 950 \\
\hline & & $\bar{\rho}$ & 2.7 & $\bar{\rho}$ & 0.95 \\
\hline & & $h_{s} \quad[\mathrm{~mm}]$ & $0.40 ; 0.79 ; 3.18^{*}$ & $h_{s} \quad[\mathrm{~mm}]$ & $0.79 ; 1.59 ; 3.18^{*}$ \\
\hline & & $\bar{h}_{s}$ & $0.005 ; 0.01 ; 0.02$ & $\bar{h}_{s}$ & $0.01 ; 0.02 ; 0.04$ \\
\hline & & $\Pi_{1}$ & $180 ; 1,430 ; 91,300 *$ & $\Pi_{1}$ & $30 ; 250 ; 2,030^{*}$ \\
\hline
\end{tabular}

* For each model the structural thicknesses are listed in order from smallest to largest for each material and $\Pi_{1}$ is listed respectively

\section{Flow Facility and Instrumentation}

The experimental facility used was the University of Michigan's closed loop, free surface water channel. The water channel's test section is 24 " x 24 " with glass sides and bottom. The water channel's freestream velocity capability ranges from $5 \mathrm{~cm} / \mathrm{s}$ to $40 \mathrm{~cm} / \mathrm{s}$. The water channel's turbulence intensity was measured using PIV and is approximately $1 \%$. The turbulence intensity includes a low frequency undulation caused by the free-surface. 
Test models are actuated by a pitch-plunge oscillator rig above the water channel's free surface. The oscillator rig consists of a B4872TS Rotary Table (pitch simulation) and a Velmex 20-inch BiSlide (plunge simulation). The two motor stages are controlled by a Velmex VXM-1-1 motor controller.

\section{A. Flowfield Measurement}

The two-dimensional PIV system is based on a double frame, single exposure design. A Quanta-Ray PIV Series Pulsed Nd:YAG laser creates a light sheet perpendicular to a charged-coupled device (CCD) array. The light sheet enters the test section side-window with the CCD array facing the bottom-window perpendicular to the light sheet and exposed to the chordwise cross section of the flat plate.

A Cooke Corporation PCO.4000 camera houses the $4008 \times 2672$ pixel CCD array. The camera provides relatively high resolution for a given focal length lens.

The seeding particles are Titanium Dioxide $\left(\mathrm{TiO}_{2}\right)$ with a particle diameter of $3 \mu \mathrm{m}$. Laser light intensity is adjusted to irradiate the particles providing the ideal particle image diameter, approximately $3 \times 3$ pixels for a $32 \times$ 32 pixel interrogation window.

Table 5 provides a summary of the relevant parameters of the PIV measurements.

Table 5. Table of PIV parameters

\begin{tabular}{|c|c|}
\hline \multicolumn{2}{|l|}{ Model } \\
\hline Chord, c [mm] & 79 \\
\hline Span, $\mathrm{b}[\mathrm{mm}]$ & 241 \\
\hline \multicolumn{2}{|l|}{ Flow } \\
\hline Fluid & Water \\
\hline $\operatorname{Re}$ & $5.3 \times 10^{3}$ \\
\hline$U_{\infty}[\mathrm{mm} / \mathrm{s}]$ & 67 \\
\hline \multicolumn{2}{|l|}{ Seeding } \\
\hline Type & $\mathrm{TiO}_{2}$ \\
\hline Particle Physical Diameter $[\mu \mathrm{m}]$ & 3 \\
\hline Dispersant & DARVAN C-N \\
\hline \multicolumn{2}{|l|}{ Laser } \\
\hline Type & $\mathrm{Nd}: Y A G$ \\
\hline Max Pulse Energy [mJ/pulse] & 200 \\
\hline \multicolumn{2}{|l|}{ Recording } \\
\hline $\mathrm{FOV}\left[\mathrm{mm}^{2}\right]$ & $\cong 17,000$ \\
\hline Camera & PCO.4000 \\
\hline Pixel Length Scale [pixels/mm] & 25 \\
\hline Dynamic Range & 16 bit \\
\hline f-number & 5 \\
\hline Focal length $[\mathrm{mm}]$ & 105 \\
\hline Particle Image Diameter [pixels] & 3 \\
\hline$\Delta t[\mathrm{~ms}]$ & 3.626 \\
\hline \multicolumn{2}{|l|}{ Image Pair Processing } \\
\hline High Resolution Window [pixels ${ }^{2}$ ] & $32 \times 32$ \\
\hline Low Resolution Window [pixels ${ }^{2}$ ] & $64 \times 64$ \\
\hline Sample Size* [min. - max.] & approx. $240-270$ \\
\hline Total PIV Realizations & 270 \\
\hline
\end{tabular}

PIV was performed at the $50 \%$ and $75 \%$ span which coincided with dye flow visualization planes. Figure 2 shows the coincident dye flow and PIV planes. The image in Figure 2 is from dye flow visualization with a superimposed green line at the $75 \%$ span location marking the coincident PIV plane. 


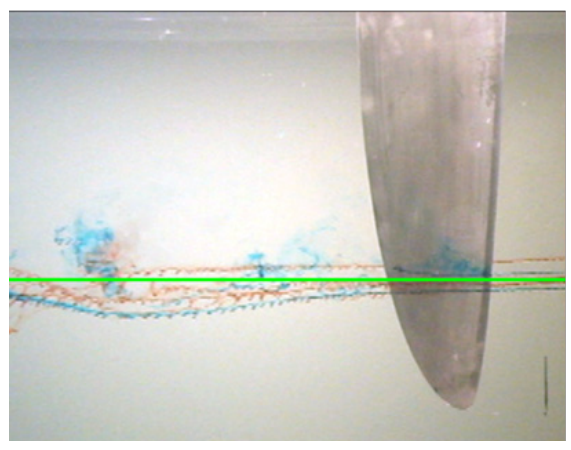

Figure 2. Coincident dye flow visualization and PIV planes (green line), flow is from left to right

\section{B. Force Measurement}

Direct force measurements were performed with an ATI industrial automation Nano43 six-axis sensor, ATI industrial automation 9105-IFPS-1 interface power supply, National Instrument PCI-6625 data acquisition card, and a desktop computer. The sensor was mounted between the pitch/plunge actuator and the wing above the free surface water channel. The force/ torque uses silicon strain gauges and measures forces up to $36 \mathrm{~N}$ in all force directions and torques up to $500 \mathrm{~N}-\mathrm{mm}$ on all torque axes. The force measurements have a $1 / 128 \mathrm{~N}$ resolution and torque measurements have a $1 / 10 \mathrm{~N}-\mathrm{mm}$ resolution.

The strain gauges are sampled at $1,500 \mathrm{~Hz}$ over 105 motion cycles after 5 seconds of pre-trigger. The high sampling rate was necessary to capture the electromagnetic interferences and other analog noise for later low-pass filtering. The first 10 cycles are removed to eliminate startup effects leaving 95 cycles for phase averaging.

The phase-averaged forces were smoothed with a multiple-pass symmetric moving average. The power and amplitude spectra of all signals were examined before smoothing to check that only noise was removed.

Given the parametric configuration of this study only the lift force is discussed and all subsequent force data including tares are lift forces. Tares were performed to ensure that the force measured was due to hydrodynamic forces on the wing. More specifically, the tare eliminated the added forces from the mounting apparatus, endplate and wing's structural mass. Figure 3 plots the comparison of the wetted endplate and wetted model tares. The wetted endplate tare is an order magnitude larger than the wetted model tare.

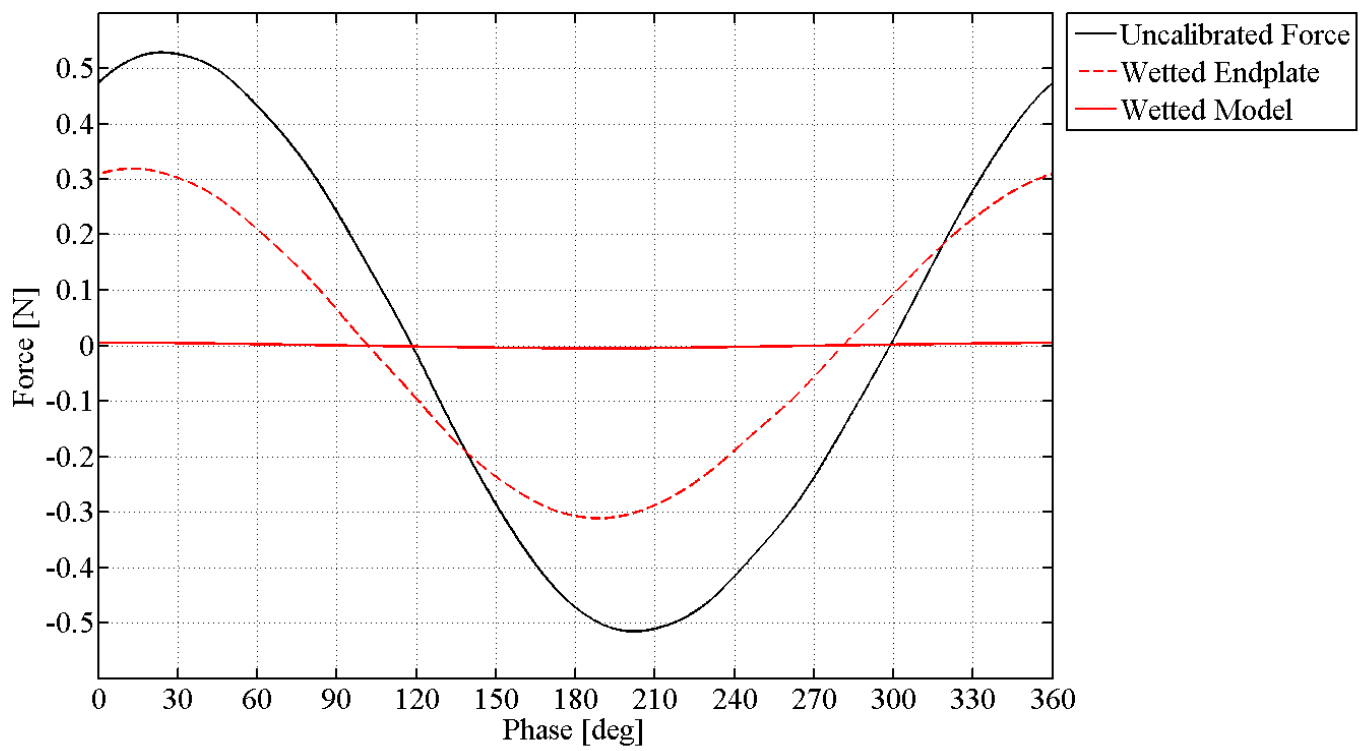

Figure 3. The uncalibrated force measurement $\left(\Pi_{1}=180\right)$ compared with the contribution of the wetted model (hydrodynamic force) and wetted model (inertial force)

The wetted endplate tare was generated with direct force measurement. The tare equals the difference between experiments with and without a model.

American Institute of Aeronautics and Astronautics 
The wetted model tare was generated with laser Doppler vibrometry. The model was sampled at eight locations described in the Structural Deformation Measurement section. For each model the measured deformations are used to calculate and then interpolate the plate acceleration along the model's span. The acceleration is then used to calculate the inertial force contribution of each model's structural mass. The vibrometer inertia tare is compared with an inertia tare using direct force measurement on each model in air in Figure 4. The air tare is inferior to the vibrometer tare because phase lags and dampening differ from the wetted experiments.
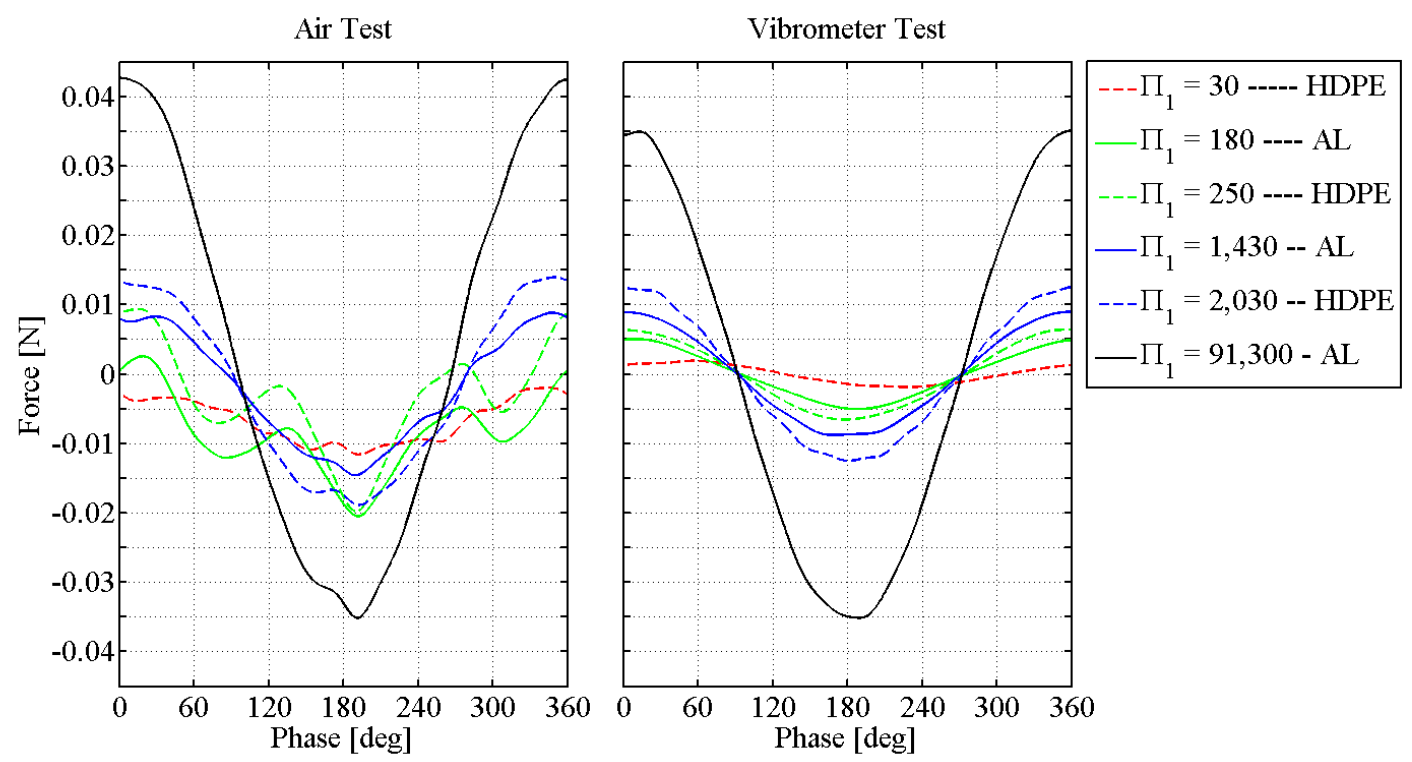

Figure 4. Comparison of two model inertia measurements; the left plot is direct force measurement of the model's inertial force contribution in air and the right plot is the indirect force measurement of the model's inertial force contribution in water with laser vibrometer

The force measurement discussion will conclude with a check of measurement accuracy. The phase averaged forces are compared with the corresponding standard deviation in Figure 5. The magnitude of the standard deviation approaches and, at times, is superior to the calibrated resolution $(\sim 0.008 \mathrm{~N})$.

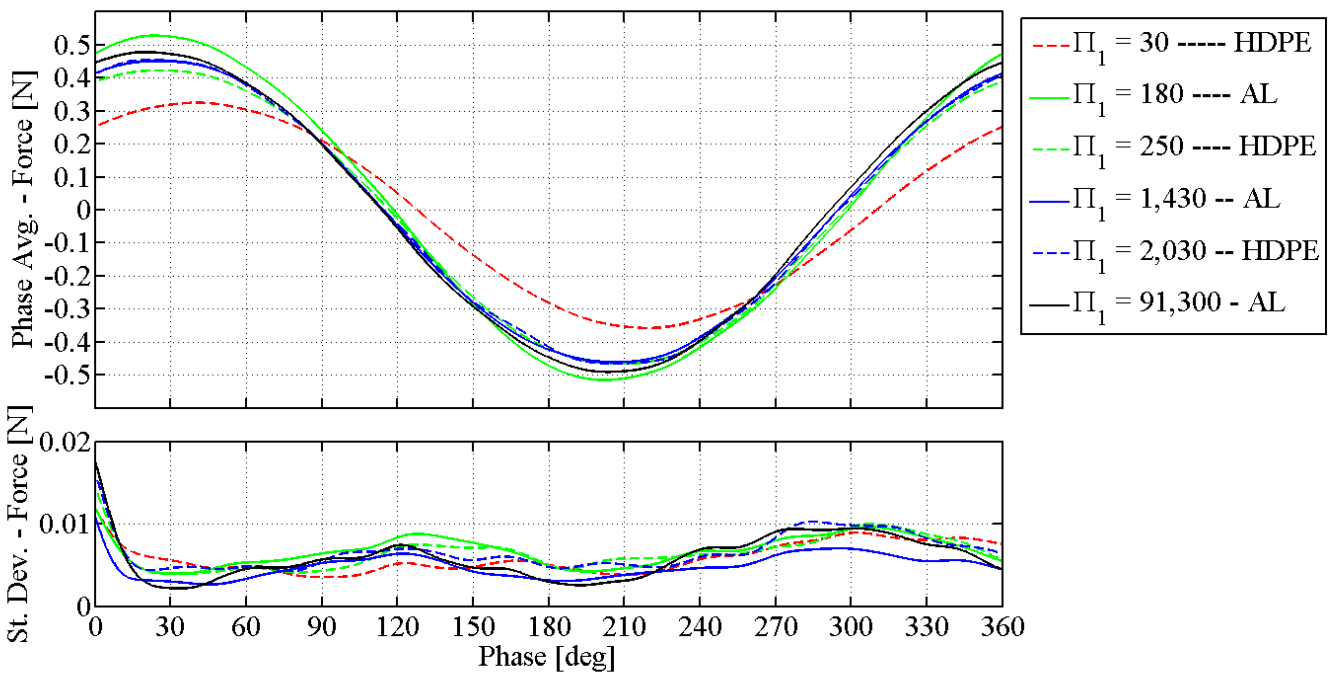

Figure 5. Uncalibrated phase averaged force and standard deviation showing measurement accuracy. 


\section{Structural Deformation Measurement}

For this flexible flapping wing experiment 'contact' measures (i.e. accelerometers) of the wing's deformation would not be appropriate; therefore laser Doppler vibrometry (LDV) was selected to quantify the structural deformation in a noninvasive manner.

The LDV measurements were performed with a Polytec PSV-400 Scanning Vibrometer ${ }^{18,19}$. The vibrometer system is composed of the PSV-I-400 laser head, the OFV-5000 controller, the PSV-E-401 junction box, and a desktop computer. The PSV-I-400 laser head uses a He-Ne laser to emit a $633 \mathrm{~nm}$ linear polarized beam. Using the principles of interferometry, the vibrometer system is able to yield velocities by measuring the interference pattern generated by the different path lengths between a reference beam and a backscattered object beam. The OFV-5000 is responsible for interpreting the interference pattern. The PSV-E-401 junction box is responsible for interfacing and synchronizing all hardware.

To facilitate a diffuse reflection of the backscattered laser light reflective tape was placed at the sampling locations; therefore, the reflective tape aides in identifying the sampling locations in Figure 6. The vibrometer measured the velocity at the quarter and three-quarter chord at the $0 \%, 25 \%, 50 \%$, and $75 \%$ span location.
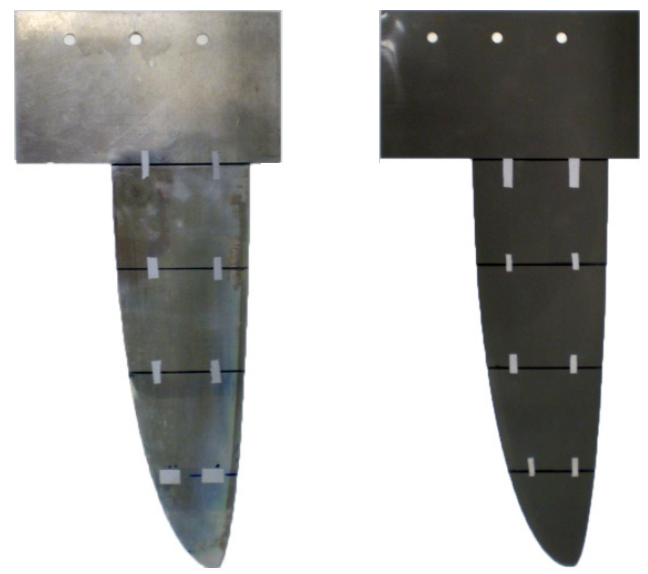

Figure 6. Aluminum (left) and HDPE (right) models with reflective tape at the eight sampling locations

Belaboring the point, Figure 7 is an example of the $75 \%$ span location where Figure $7(a)$ is the three-quarter chord sampling location and Figure 7(b) is the quarter chord sampling location. The images in Figure 7 are important for they are from the camera onboard the PSV-I-400 laser head that allows the sampling location to be known within a pixel on the image.

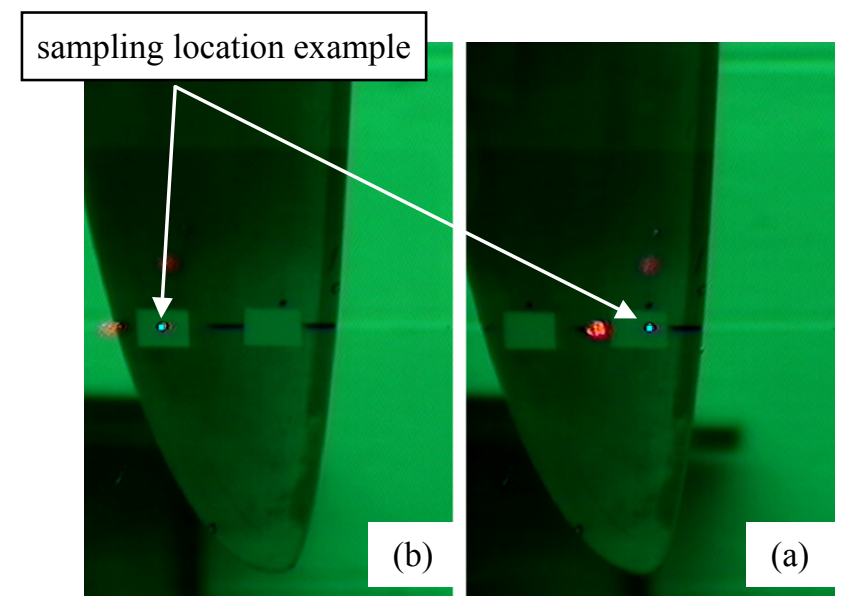

Figure 7. Laser vibrometer sampling locations with freestream flowing from, (a) is the quarter chord location at the $75 \%$ span location, (b) is the three-quarter chord location at the $75 \%$ span location 
Data was acquired over 30 periods of motion with the first 5 being discarded because of startup effects. The velocity is sampled at $512 \mathrm{~Hz}$. The velocity data is interpolated and partitioned into 25 arrays. Each of the 25 interpolated arrays is numerically integrated to yield 25 displacement arrays. Both the velocity and displacement arrays are phase-averaged. The phase-averaged signals were smoothed with a multiple-pass symmetric moving average with three taps. The power spectra of all the acquired signals were examined before smoothing to check that significant signals weren't excluded.

Since the backscattered laser light is passing through two mediums (water and air) a correction is necessary. A 0.75 scaling factor on the velocity measurement is used the correct for the mismatched indices of refraction.

\section{Results and Discussion}

The effect of flexibility has previously been studied using the same planform and forward flight condition. The current study builds on the past work by adding two models in a $\Pi_{1}$-parameter range of interest and introducing force measurement. The results presentation begins with an overview of the structural deformations and resulting effective angle of attack then continues with force production and PIV results.

\section{A. Structural Deformation}

The structural deformation was measured with a one-component LDV. The normalized plunge amplitude was measured at the 75\%-span location and plotted in Figure 8 as a function of motion phase. Figure 8 illustrates the effect of decreasing the $\Pi_{1}$-parameter on the deformation. To clarify, if the plotted variable in Figure 8 oscillates with amplitude of 1 then the entire wing rigidly follows the wing root's motion. If the plotted variable in Figure 8 oscillates with amplitude other than 1 then the wing is structurally deforming and may lag or lead the wing root motion. For the models listed in order of decreasing $\Pi_{1}$ the measured plunge amplitude at $75 \%$ span in Figure 8 differed from the root by $1.6 \% .0 .4 \%, 1.7 \%, 7.7 \%, 23.1 \%$, and $43.4 \%$.

The deformations in Figure 8 are reported at the $75 \%$-span with no regard to the chordwise location because of the negligible twist discovered in Rausch, et al. ${ }^{8}$ and therefore deformations are reported from the $0.25 \%$-chord location for the remainder of the section.

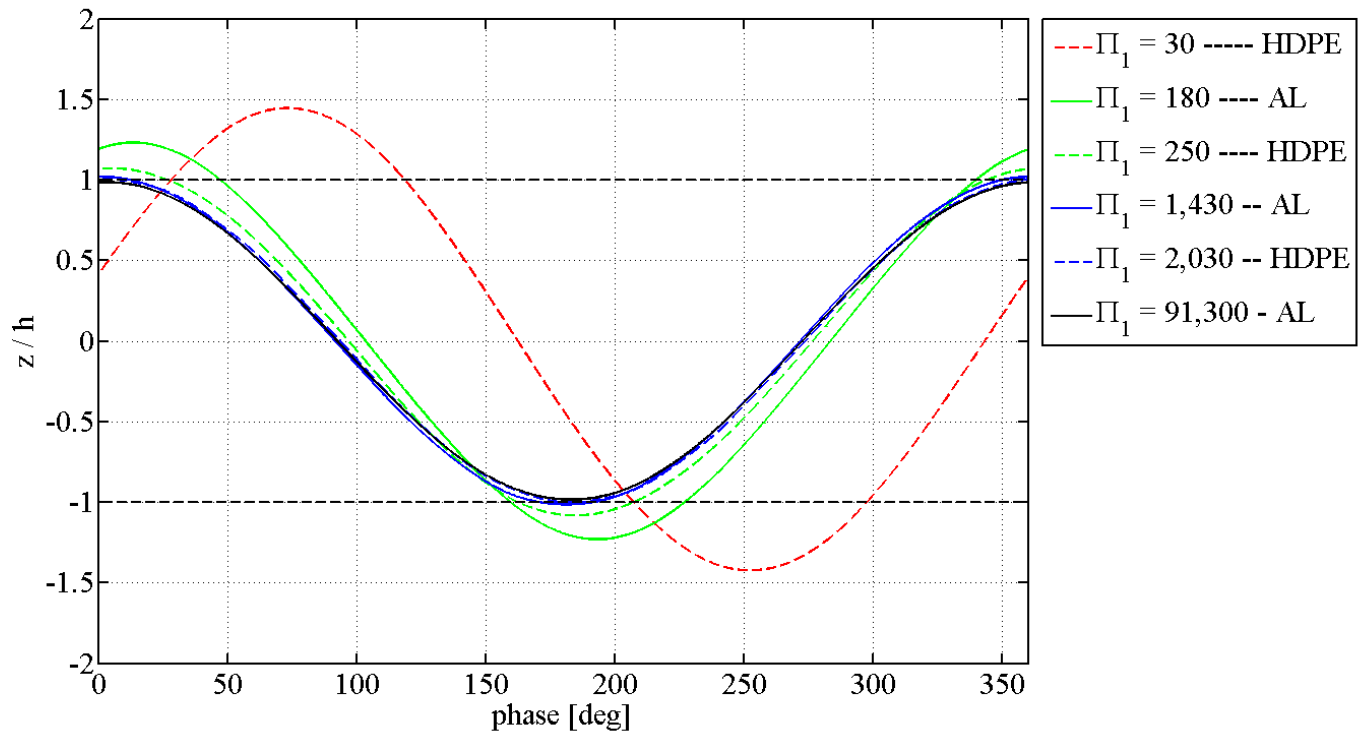

Figure 8. Reduced plunge amplitude at 75\%-span for all six wings

In addition to the deformations there are phase lags present in the reduced plunge amplitude plot in Figure 8 with force production significance. Heathcote, et al. ${ }^{9}$. found that deformations that remained in phase with wing root produced greater force than the rigid condition while deformations that lag the wing root are detrimental to force production. Table 6 summarizes the phase lags observed in Figure 8. The lags were large with increased flexibility and nominally yielded the root phase for $\Pi_{1} \geq 1,430$. 
Table 6. Phase lags between the maximum positive reduced amplitudes of oscillation at the root and the 75\% span location

\begin{tabular}{cccccccc}
\hline & root phase & $\Pi_{1}=30$ & $\Pi_{1}=180$ & $\Pi_{1}=250$ & $\Pi_{1}=1,430$ & $\Pi_{1}=2,030$ & $\Pi_{1}=91,300$ \\
\hline root phase & $0^{\circ}$ & $73.2^{\circ}$ & $13.5^{\circ}$ & $5.0^{\circ}$ & $1.1^{\circ}$ & $2.7^{\circ}$ & $2.8^{\circ}$ \\
\hline \hline
\end{tabular}

\section{B. Effective Angle of Attack}

Effective angle of attack has been shown to be an important parameter for studying the flow topology and force production of the unsteady flows generated by pitching and plunging airfoils ${ }^{20}$.

The rigid wing kinematics of this study generates a sinusoidal plunge-induced angle of attack profile with a $32.5^{\circ}$ amplitude of oscillation. The structural deformations in Figure 8 indicated that with increased flexibility the plunge amplitude increased farther from the root while maintaining the same oscillation frequency. The increased plunge amplitude at the same frequency of oscillation yields an effective angle of attack time history larger than the plunge-induced angle of attack. Continuing with the $75 \%$-span location, the effective angle of attack time history is plotted in Figure 9 illustrating the increased effective angle of attack amplitude; additionally, a phase lag appears with greater flexibility.

For the models listed in order of decreasing $\Pi_{1}$ the measured effective angle of attack at $75 \%$ span is $-0.1 \%$. $3.4 \%, 2.2 \%, 7.6 \%, 12.1 \%$, and $30.8 . \%$ greater than the $32.5^{\circ}$ prescribed at the root.

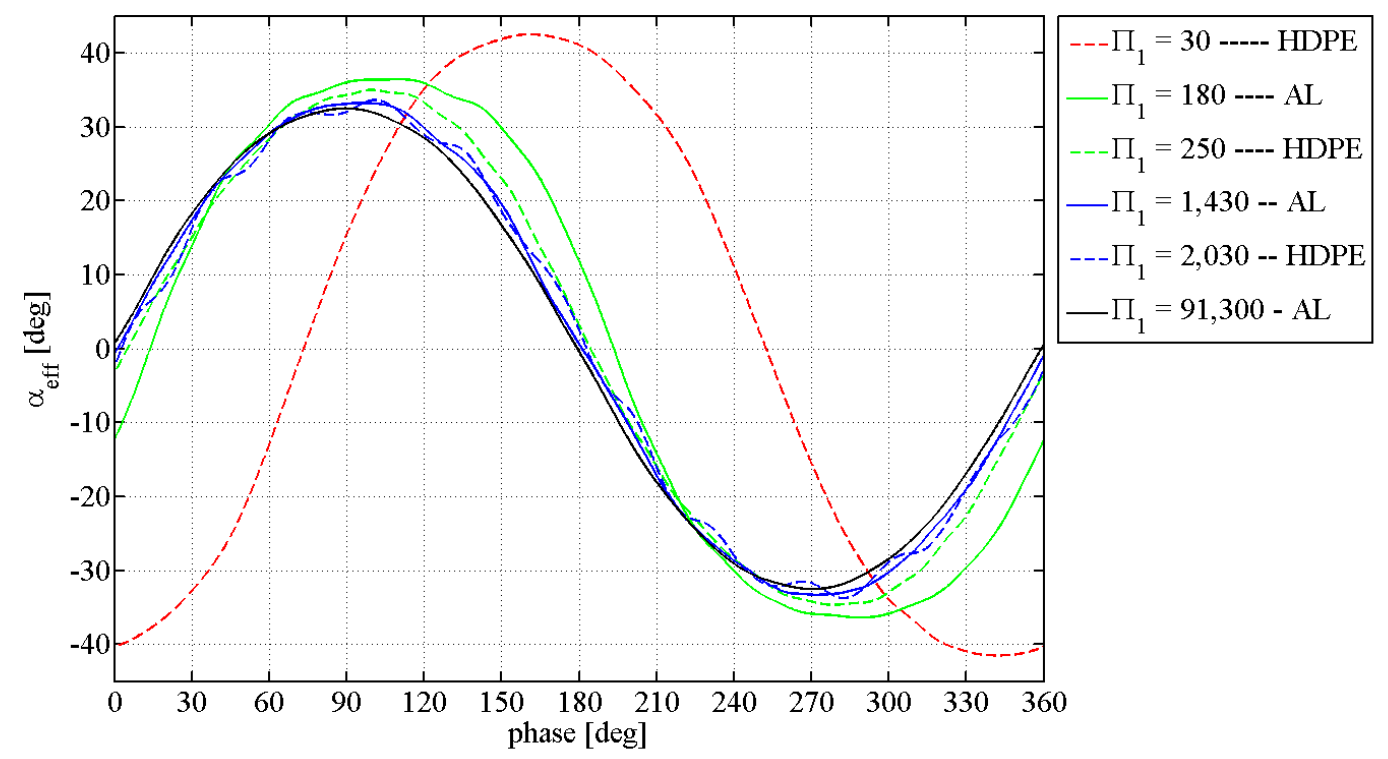

Figure 9. Vibrometer measured effective angle of attack at 75\%-span.

Similar to the deformation measurement, the effective angle of attack profiles have phase lags that are summarized in Figure 11.

Table 7. Phase lags between the maximum effective angle of attack at the root and the $75 \%$ span location

\begin{tabular}{cccccccc}
\hline \hline & root phase & $\Pi_{1}=30$ & $\Pi_{1}=180$ & $\Pi_{1}=250$ & $\Pi_{1}=1,430$ & $\Pi_{1}=2,030$ & $\Pi_{1}=91,300$ \\
\hline root phase & $0^{\circ}$ & $70.7^{\circ}$ & $20.1^{\circ}$ & $9.6^{\circ}$ & $5.0^{\circ}$ & $10.8^{\circ}$ & $-0.4^{\circ}$ \\
\hline \hline
\end{tabular}

\section{Force Production}

Forces were measured per the aforementioned procedure and lift generation in excess of theoretical models was of paramount interest. Thrust was ignored because of the lack of geometric angle of attack, pure-plunge motion kinematics, small cross-sectional thickness, and lack of aerodynamic twist.

The total lift force was compared to the quasi-steady and unsteady form of the Theodorsen ${ }^{21}$ model for pure plunge motion kinematics. The Theodorsen model is relevant because it provides a model for the lag between fluid dynamic response and the motion kinematics. The Theodorsen model is given in Equation 1 for lift per unit span. 


$$
C_{l}(t)=\frac{\pi c}{2} \frac{\ddot{h}}{U_{\infty}^{2}}+2 \pi C(k) \frac{\dot{h}}{U_{\infty}}
$$

The Theodorsen model in Equation 1 uses a two-dimensional incompressible inviscid flow model for a plunging thin airfoil, planar wake, and tailing-edge Kutta condition. The contributions to the lift coefficient in Equation 1 can be attributed to a circulatory forces (function of effective angle of attack and reduced frequency) and non-circulatory force (inertia).

The comparison with the experimental results requires strip theory to compute the total lift coefficient on the theoretical wing. The closed form solution for total lift coefficient was derived for the elliptical wing in Equation 2.

$$
C_{L}(t)=\frac{4 c}{3} \frac{\ddot{h}}{U_{\infty}^{2}}+2 \pi C(k) \frac{\dot{h}}{U_{\infty}}
$$

Using the results of Equation 2, a breakdown of the theoretical model is plotted in Figure 10. The noncirculatory and circulatory components are more beneficially in-phase for the quasi-steady model than the unsteady model resulting in the quasi-steady model producing greater force.

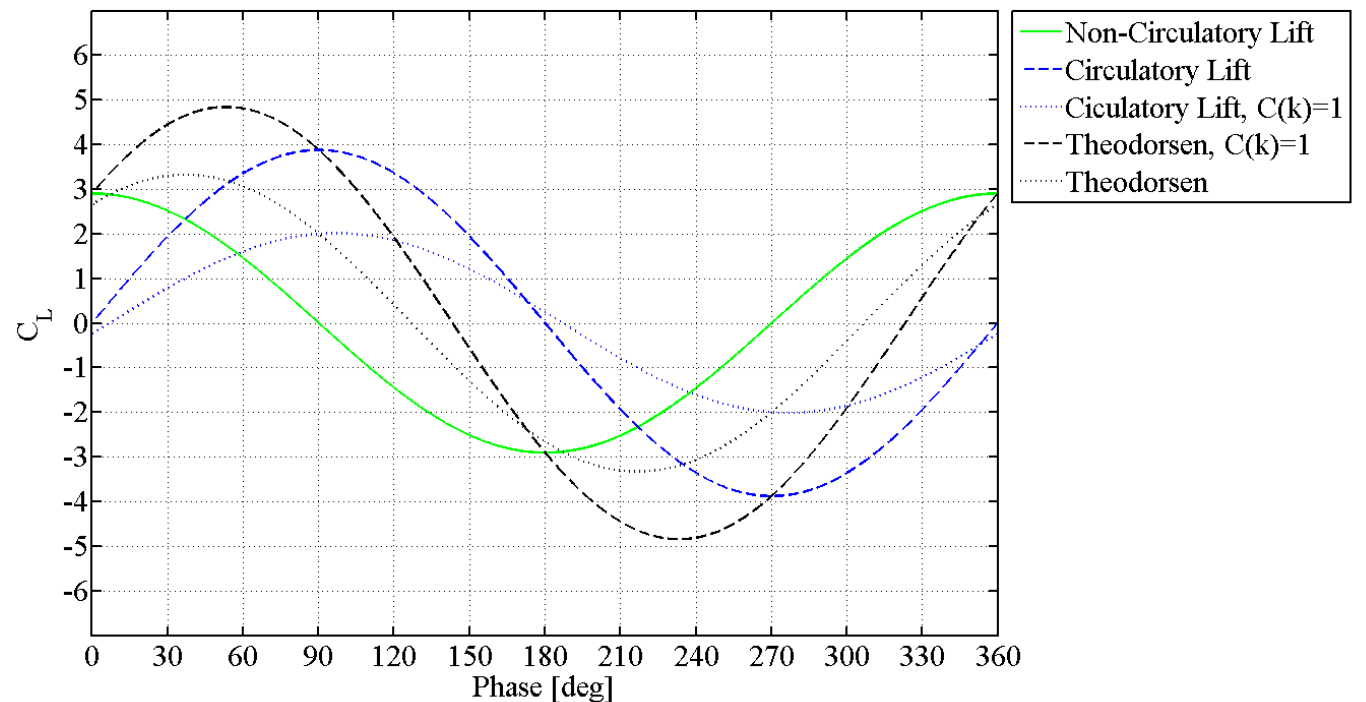

Figure 10. Breakdown of non-circulatory and circulatory contributions to the quasi-steady $(C(k)=1)$ and unsteady Theodorsen models

The quasi-steady and unsteady theoretical models are now combined with the experimental results as a function of the motion phase in Figure 11. The quasi-steady theoretical model is in good agreement with the nominally rigid models and the unsteady theoretical model is in poor agreement with all experimental results.

The moderately $\left(\Pi_{1}=180\right)$ and highly flexible $\left(\Pi_{1}=30\right)$ wings yield significantly different force histories. The moderately flexible wing produced the greatest amount of force while staying nearly in-phase with the rigid motion. The highly flexible wing produced the least amount of force and significantly lagged the other experimental and theoretical results. 


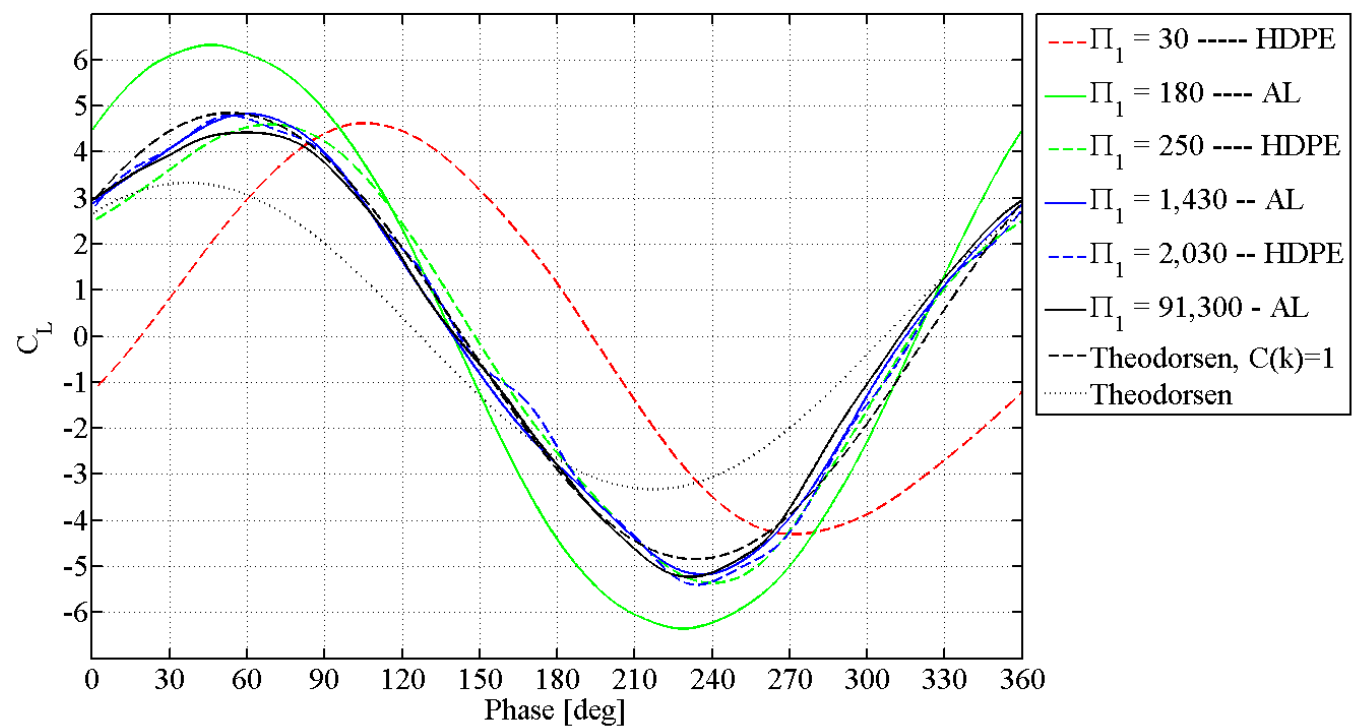

Figure 11. Wing lift coefficient as a function of motion phase is plotted for the six wings and the quasi-steady and unsteady form of the Theodorsen model

The $C_{L}$-min, $C_{L}$-max, and $C_{L}$-range in Figure 11 are listed in Table 8. The moderately flexible wing has the largest $C_{L}$ production with -6.4 $C_{L}$-min and $6.3 C_{L}$-max. The highly flexible wing has the smallest $C_{L}$ production with $-4.3 C_{L^{-}}$ min and $4.6 C_{L}$-max.

Table 8. For each curve in Figure 8 the minimum, maximum, and corresponding range of lift coefficient

\begin{tabular}{ccccccccc}
\hline \hline & $\Pi_{1}=30$ & $\Pi_{1}=180$ & $\Pi_{1}=250$ & $\Pi_{1}=1,430$ & $\Pi_{1}=2,030$ & $\Pi_{1}=91,300$ & $\begin{array}{c}\text { Theod., } \\
\mathrm{C}(\mathrm{k})=1\end{array}$ & Theod. \\
\hline$C_{L}$-Min & -4.3 & -6.4 & -5.4 & -5.2 & -5.4 & -5.2 & -4.9 & -3.3 \\
$C_{L}$-Max & 4.6 & 6.3 & 4.6 & 4.8 & 4.8 & 4.4 & 4.9 & 3.3 \\
$C_{L}$-Range & 8.9 & 12.7 & 10.0 & 10.0 & 10.2 & 9.7 & 9.7 & 6.7 \\
\hline \hline
\end{tabular}

Similar to the structural deformation measurements, phase lags were observed in the force data. The phase lags are reported from the root's phase at maximum displacement to the maximum $C_{L}$ 's phase in Table 9.

The phase lags for nominally rigid wings are in the range from $53.3^{\circ}-60.0^{\circ}$. This range agrees with the quasisteady theoretical model's phase of 53.2. The moderately flexible wings have phase lags in the range $45.9^{\circ}-69.2^{\circ}$. The $45.9^{\circ}$ lag corresponds to the $\Pi_{1}=180$ model and its maximum lift coefficient leads that of the rigid wings and the quasi-steady theoretical model. The highly flexible wings has a phase lag of $105.1^{\circ}$. This is the largest phase lag and corresponds to the worst force production.

Table 9. Lags between the root's maximum displacement and each wing's peak positive total lift coefficient

\begin{tabular}{cccccccccc}
\hline & root & $\Pi_{1}=30$ & $\Pi_{1}=180$ & $\Pi_{1}=250$ & $\Pi_{1}=1,430$ & $\Pi_{1}=2,030$ & $\Pi_{1}=91,300$ & $\begin{array}{c}\text { Theod. } \\
\mathrm{C}(\mathrm{k})=1\end{array}$ & Theod. \\
\hline root & $0^{\circ}$ & $105.1^{\circ}$ & $45.9^{\circ}$ & $69.2^{\circ}$ & $60.0^{\circ}$ & $53.3^{\circ}$ & $60.1^{\circ}$ & $53.2^{\circ}$ & $36.9^{\circ}$ \\
\hline \hline
\end{tabular}

\section{PIV Results}

The PIV results for $\Pi_{1}=180$ are presented in Figures 13 and 14 for the downstroke and upstroke, respectively. The PIV results for $\Pi_{1}=250$ are presented in Figures 15 and 16, also for the downstroke and upstroke, respectively. Each figure shows vorticity contours and streramlines at different phases at $50 \%$ and $75 \%$ span locations. These figures are similar to the cases reported by Rausch, et al.. Leading edge (LEV) and trailing edge (TEV) vortices form on the top surface during the downstroke. The LEV remains attached at the end of the downstroke and interacts with the plate during the upstroke. The TEV sheds into the wake that for these conditions is not flap. The vorticity contours show multiple vortex cores form at the trailing edge during the downstroke. Streamlines during the 
downstroke show a closed recirculation region, with incoming streamlines terminating on the airfoil. During the upstroke the streamlines starting at the airfoil are opened on the downstream side of the flow

For the moderately flexible wings the $50 \%$ and $75 \%$ span locations were analyzed for differences in the leading edge vortex size and phase because of the LEV role in force production. The $\Pi_{1}=180$ wing produced a larger vortex at the $75 \%$ span location due to the increased effective angle of attack; however, in contrast with the highly flexible wing the LEV at $50 \%$ span does not significantly lag the LEV at $75 \%$ span. The $\Pi_{1}=250$ wing produced approximately the same size LEV at both span locations due to the more similar effective angle of attack and the small variation in chord. Additionally, for this wing the LEV at 50\% span lags $75 \%$ span. 
$0^{\circ}$
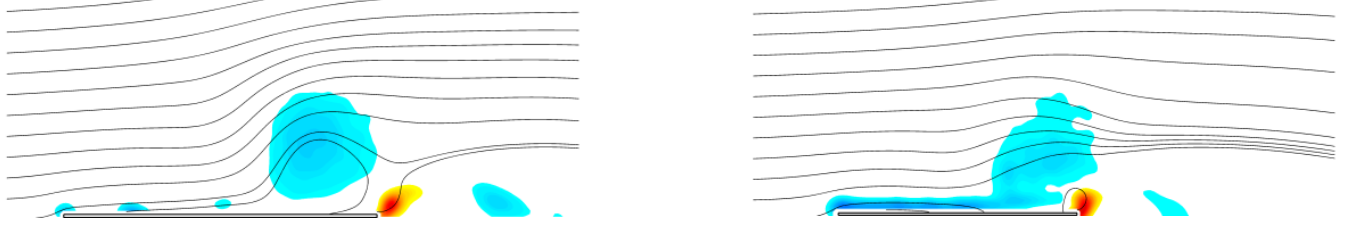

$30^{\circ}$
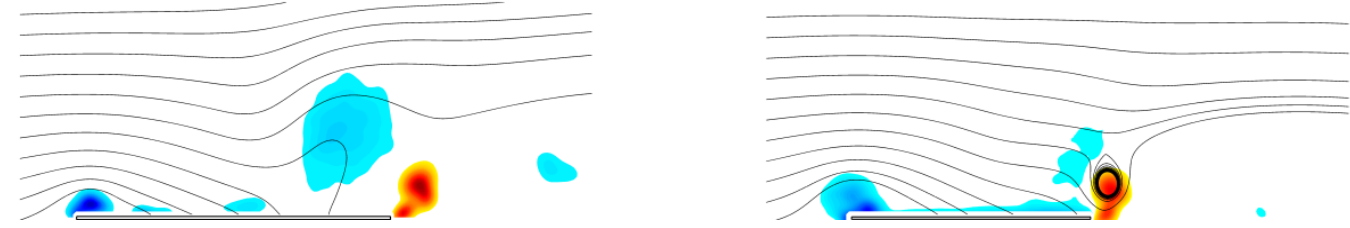

$60^{\circ}$
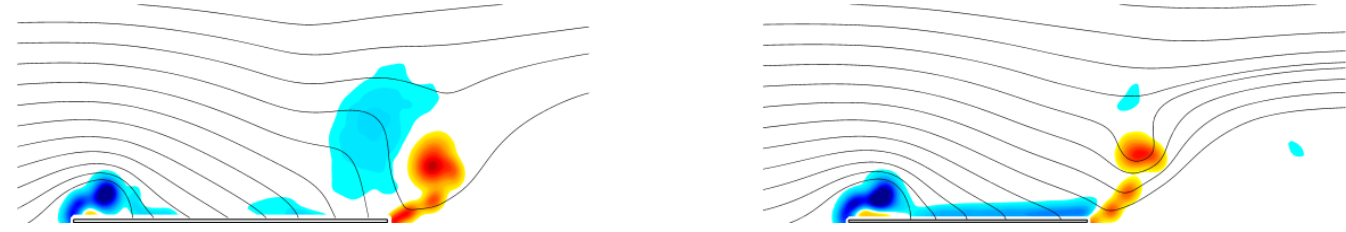

$90^{\circ}$
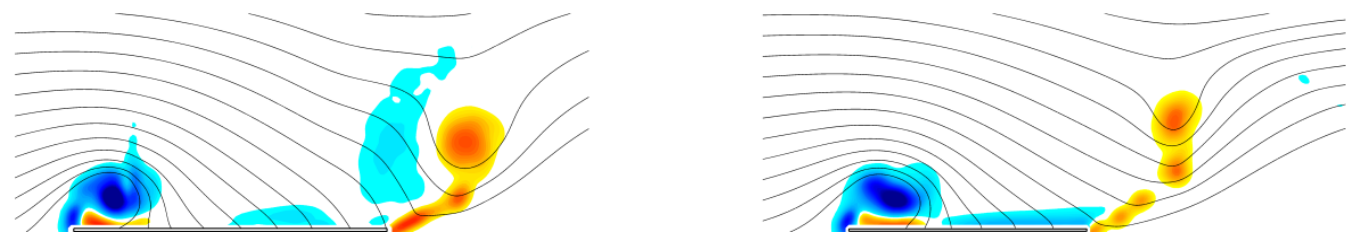

$120^{\circ}$
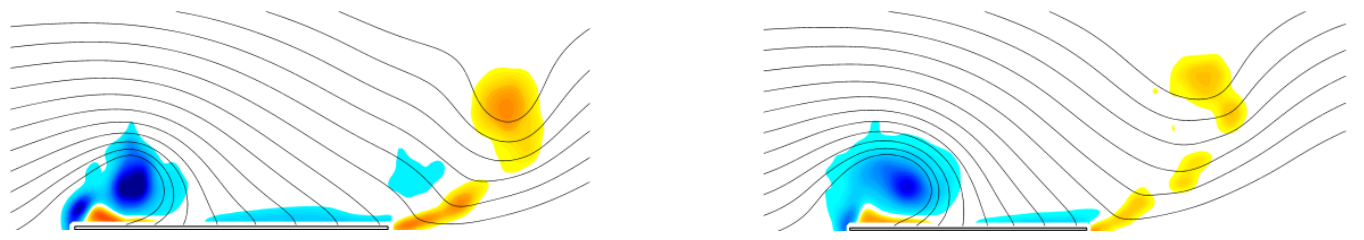

$150^{\circ}$

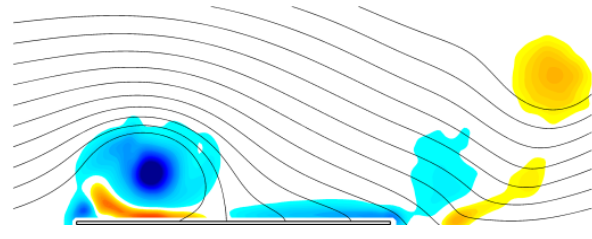

$\omega^{*} @$, 50\%-span

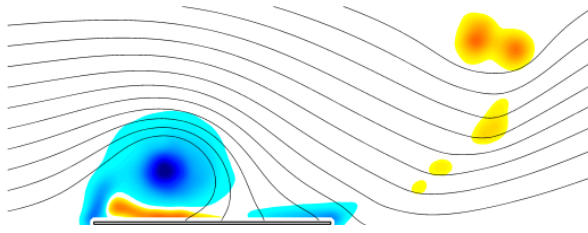

$\omega^{*} @$ (75\%-span

Figure 12. Normalized vorticity contour plots for a wing with moderate flexibility, $\Pi_{1}=180$. The contour plots follow the downstroke from $0^{\circ}-150^{\circ}$ showing streamline curvature forming a closed separation caused by the leading edge vortex 


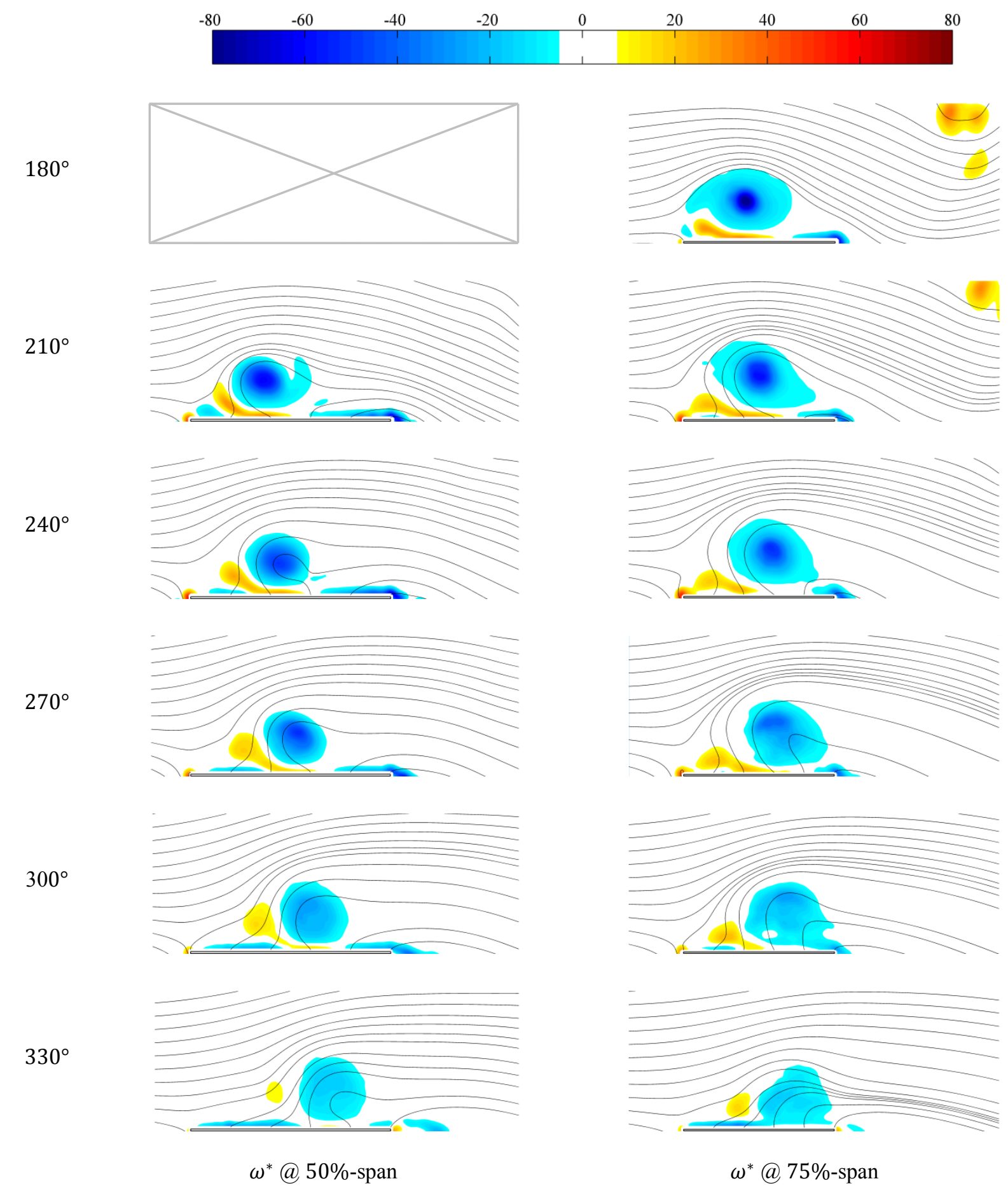

Figure 13. Normalized vorticity contour plots for a wing with moderate flexibility, $\Pi_{1}=180$. The contour plots follow the upstroke from $180^{\circ}-330^{\circ}$ showing the interaction of the leading edge vortex with the plate. 
$0^{\circ}$

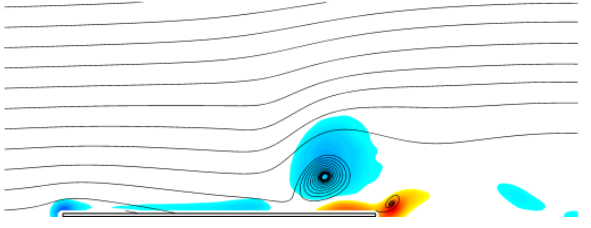

$30^{\circ}$

$60^{\circ}$

$90^{\circ}$
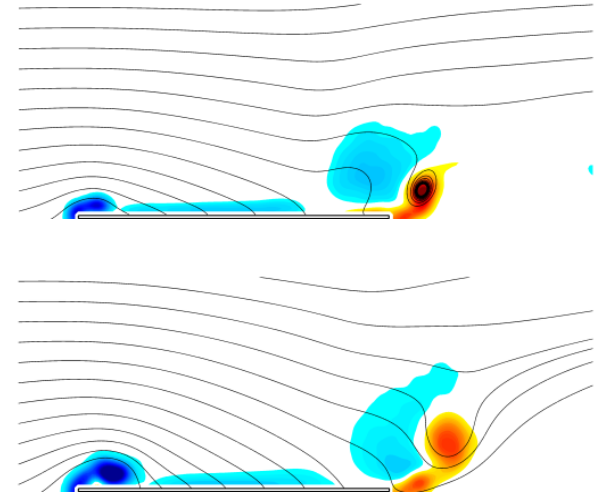

$120^{\circ}$

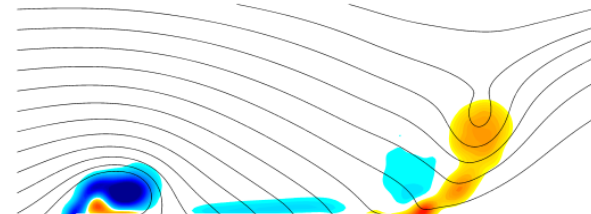

$150^{\circ}$
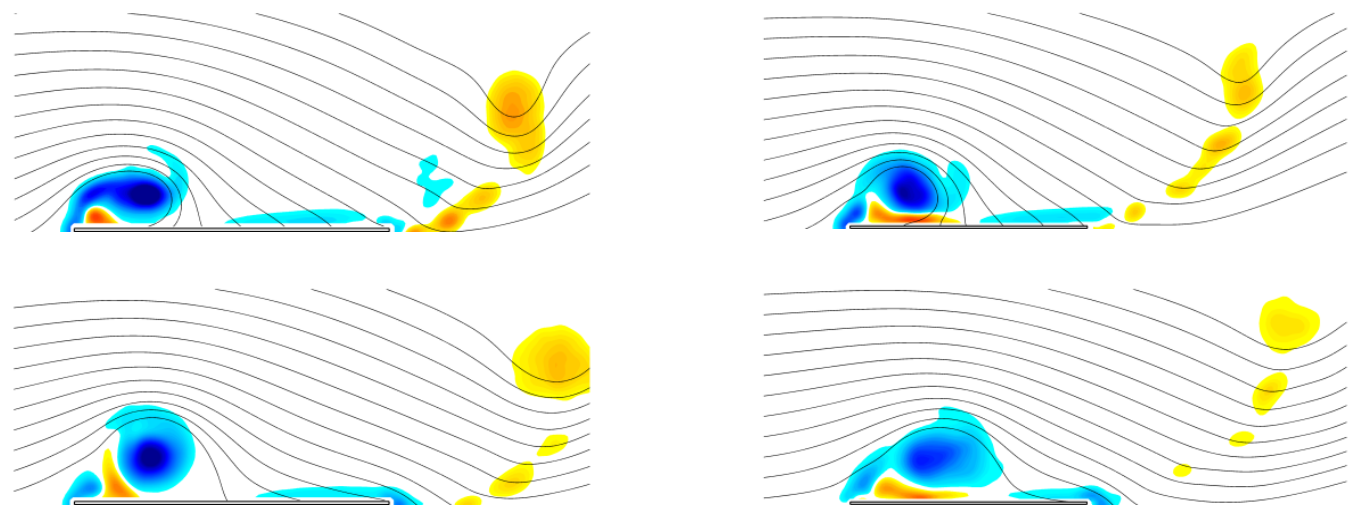

$\omega^{*} @ 50 \%-\operatorname{span}$
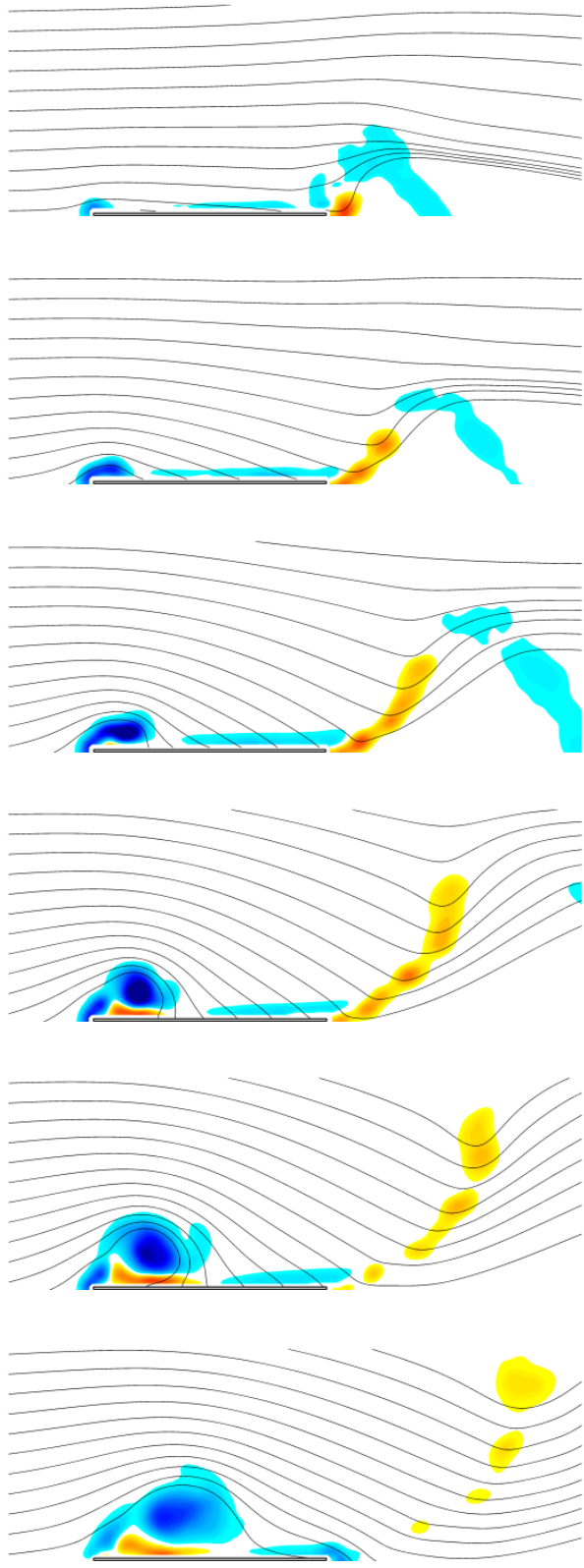

$\omega^{*} @ 75 \%$-span

Figure 14. Normalized vorticity contour plots for a wing with moderate flexibility, $\Pi_{1}=250$. The contour plots follow the downstroke from $0^{\circ}-150^{\circ}$ showing streamline curvature forming a closed separation caused by the leading edge vortex 


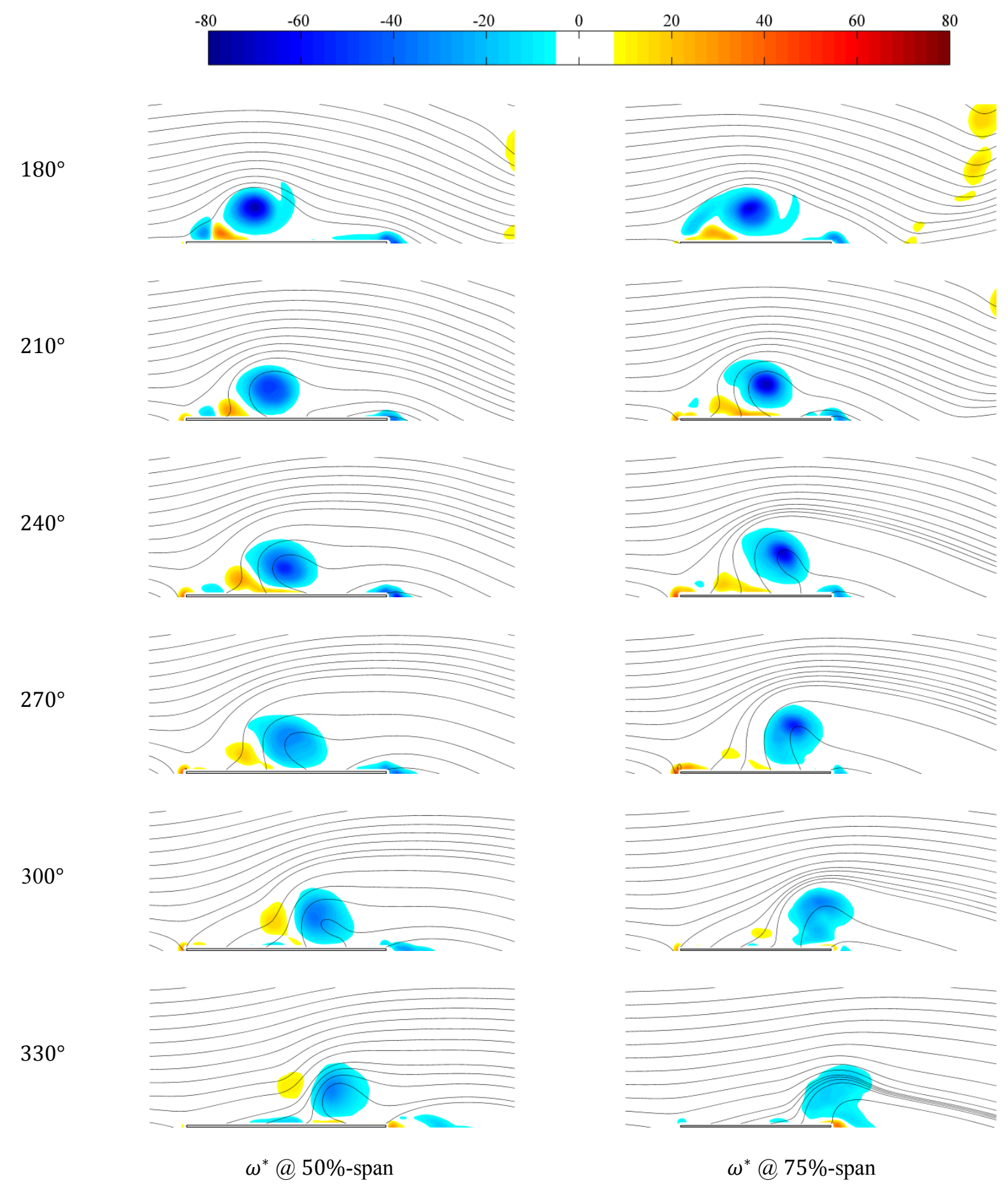

Figure 15. Normalized vorticity contour plots for a wing with moderate flexibility, $\Pi_{1}=250$. The contour plots follow the downstroke from $180^{\circ}-330^{\circ}$ showing the interaction of the leading edge vortex with the airfoil 


\section{Conclusion}

Six models were investigated with a range of $\Pi_{1}$ covering four orders of magnitude from $10^{1}$ to $10^{4}$. The flow field, structural deformation, and forces were quantified with PIV (with dye flow visualization as a qualitative measure), laser Doppler vibrometry, and force/torque sensor, respectively. Flexibility effects are found only for values of $\Pi_{1}$ less than $10^{3}$ order of magnitude; $\Pi_{1}$ values of $10^{3}$ order of magnitude and higher did not produce significant deformation, flow topology, or force changes.

The experimental measurements indicated that the main effects of introducing flexibility for the prescribed planform shape and aspect ratio are:

- The structural deformation measurement showed:

$>$ The moderately and highly flexible wings deform in the first bending mode with no twist of fluid dynamic significance. This deformation results in a larger effective angle of attack at outboard span locations. Then the effective angle of attack histories lag the root when the wings deform.

$>$ At $75 \%$ span the plunge amplitude increased by $7.7 \%, 23.1 \%$, and $43.4 \%$ for the $\Pi_{1}=250,180$, and 30 , respectively.

- The lift force measurement showed:

$>$ The $\Pi_{1}=180$ wing produces the largest lift coefficient history $(-6.4,6.3)$ while the $\Pi_{1}=30$ wing produces the smallest lift coefficient history $(-4.3,4.6)$. All of the remaining wings produce a lift coefficient history that is in good agreement with the quasi-steady Theodorsen model $(-4.9,4.9)$.

$>$ It was observed that in addition to producing the greatest lift coefficient history, the $\Pi_{1}=180$ wing's lift force history led in phase the wings in agreement with the quasi-steady model. The $\Pi_{1}$ $=30$ wing significantly lagged the wings in agreement with the quasi-steady model thus indicating too much flexibility is detrimental to force production.

$>$ The good agreement between the quasi-steady Theodorsen model and wings with $\Pi_{1} \geq 250$ is an indication that three-dimensional effects are small and that the shed vorticity has little impact on the lift production.

- The flowfield measurement showed:

$>$ The flow topology for the moderately flexible wings is dominated by a persistent LEV presence on the suction and pressure side of the plate. The LEV on the suction side grows in circulation while the LEV on the pressure side sheds and convects downstream.

$>$ Flexibility effects are found only for values of $\Pi_{1}$ less than $10^{3}$ order of magnitude, $\Pi_{1}$ values of $10^{3}$ order of magnitude and higher did not produce significant deformation, flow topology, or force changes

\section{Acknowledgments}

This work has been supported in part by the Air Force Office of Scientific Research's Multidisciplinary University Research Initiative (MURI) and by the Michigan/AFRL (Air Force Research Laboratory)/Boeing Collaborative Center in Aeronautical Sciences.

\section{References}

${ }^{1}$ Shyy, W., Berg, M., and Ljungqvist, D., "Flapping and Flexible Wings for Biological and Micro Air Vehicles," Progress in Aerospace Sciences, Vol. 24, No. 5, 1999, pp. 455-505.

${ }^{2}$ Shyy, W., Lian, Y., Tang, J., Viieru, D., \& Liu, H. Aerodynamics of Low Reynolds Number Flyers. Cambridge University Press, New York, 2008.

${ }^{3}$ Heathcote S, Gursul I., "Flexible Flapping Airfoil Propulsion at Low Reynolds Numbers" AIAA Journal, 2007; 45(5):1066-79.

${ }^{4}$ Zhao, L., Huang, Q., Deng, X., and Sane, S., “Aerodynamic Effects of Flexibility in Flapping Wings,” Journal of the Royal Society Interface, Vol. 7. No. 44, 2010, pp. 485-497.

${ }^{5}$ Shyy, W., Aono, H., Chimakurthi, S.K., Trizila, P., Kang, C.-K., Cesnik, C.E.S. \& Liu, H., "Recent progress in flapping wing aerodynamics and aeroelasticity," Progress in Aerospace Sciences, Vol. 43, No. 7, 2010, pp 284-327.

${ }^{6}$ Ishihara, D., Horie, T., and Denda, M., "A Two-dimensional Computational Study on the Fluid-Structure Interaction Cause of Wing Pitch Changes in Dipteran Flapping Wing," The Journal of Experimental Biology, Vol. 212, 2009, pp. $3882-3891$.

${ }^{7}$ Gopalakrishnan, P., and Tafti, D. K, "Effect of Wing Flexibility on Lift and Thrust Production in Flapping Flight," AIAA Journal, Vol. 48, No. 5, 2010, pp. 865-877.

${ }^{8}$ Katz, J. and Weihs, D., "Hydrodynamic Propulsion by Large Amplitude Oscillation of an Airfoil with Chordwise Flexibility," Journal of Fluid Mechanics, Vol. 88, No. 3, 1978, pp. 485-97.

American Institute of Aeronautics and Astronautics 
${ }^{9}$ Heathcote S, Wang Z, Gursul I., "Effect of Spanwise Flexibility on Flapping Wing Propulsion," Journal of Fluids and Structures, 2008; 24:183-99.

${ }^{10}$ Chimakurthi, S. K., Tang, J., Palacios, R., Cesnik, C. E. S., and Shyy W., "Computational Aeroelasticity Framework for Analyzing Flapping Wing Micro Air Vehicles,” AIAA Journal, Vol. 47, No. 8, 2009, pp. 1865-1878.

${ }^{11}$ Heathcote S, Martin D, Gursul I., "Flexible Flapping Airfoil Propulsion at Zero Freestream Velocity," AIAA Journal, 2004;42(11):2196-204.

12 Rausch, J.M., Baik, Y., Bernal, L.P., Shyy, W., Ol, M., Cesnik, C., "Fluid Dynamics of Rigid and Spanwise-Flexible Elliptical Flat Plates at Low Reynolds Numbers," AIAA-2010-4739.

${ }^{13}$ Granlund, K., Ol, M., Garmann, D., Visbal, M., Bernal, L.P., "Experiments and Computations on Abstraction of Perching," AIAA-2010-4943

${ }^{14}$ Baik, Y., Aono, H., Rausch, J.M., Bernal, L.P., Shyy, W., Ol, M., "Experimental Study of Impulsively Rotated Flat Plate at Low Reynolds Number," AIAA-2010-4462.

15 Anderson, J. D., Fundamentals of Aerodynamics, 4th ed., McGraw Hill Higher Education, Boston, 2007.

${ }^{16}$ Kang, C.-K., Aono, H., Cesnik, C.S., Shyy, W., "A Scaling Parameter for the Thrust Generation of Flapping Flexible Wings," AIAA-2010-1313

17 Taylor, G. K., Nudds, R. L., and Thomas, A. L. R., "Flying and Swimming Animals Cruise at a Strouhal Number Tuned for High Power Efficiency," Nature, Vol. 425, No. 6959, pp. 707-711.

${ }^{18}$ Polytec Scanning Laser Vibrometer PSV 400 Hardware Manual, Polytec GmbH, 2007.

${ }^{19}$ Salas, K. I., Cesnik, C. E. S., "Guided Wave Structural Health Monitoring Using CLoVER Transducer in Composite Plates," AIAA 2009-2106.

${ }^{20}$ Baik, Y., Unsteady Force Generation and Vortex Dynamics of Pitching and Plunging Airfoils at Low Reynolds Number. Ph.D. thesis, University of Michigan, Ann Arbor, MI.

${ }^{21}$ Theodorsen, T., "General Theory of Aerodynamic Instability and the Mechanism of Flutter," N.A.C.A. Report 496, 1935 